IOSR Journal of Engineering

e-ISSN: 2250-3021, p-ISSN: 2278-8719

Vol. 2, Issue 12 (Dec. 2012), ||V4|| PP 08-14

\title{
Microcontroller Based Wireless Transmission on Biomedical Signal and Simulation in MATLAB
}

\author{
Nisha singh ${ }^{1}$, Sr. Asst. Prof. Ravi Mishra ${ }^{2}$, \\ Department of Electrical and Electronics Engineering, Shri Shankaracharya College of Engineering \& \\ Technology. jaunwani Bhilai (C.G.)
}

\begin{abstract}
Develop a simple wireless transmission system for the transmission of biomedical signal for the hospital use where we can monitor the biomedical signal like ECG, Blood pressure, Heart beats of all patients in centralized manner and we can record the ECG signals and Heart beats of all patients in a single computer. These biomedical signals are acquired and then processed with a microcontroller. After processing, all data are sent to a communication interface that can send this information to a personal computer. For the patient suffering from the cardiac disease it is very important to perform accurate and quick diagnosis. For this purpose a continuous monitoring of the ECG signal, patient's current heart rate and BP are necessary. This study covers the wireless telemedicine applications with the use of Bluetooth technology, So with the help of Bluetooth transmission and reception we can monitor the ECG signal for the lot of patient's in the central place in any hospital. In this work we also simulate the ECG waveform using MATLAB. The task of electrocardiogram (ECG) processing is based on R-peaks detection.
\end{abstract}

Keywords:- Instrumentation amplifier, Filter, Microcontroller, Bluetooth, MATLAB

\section{INTRODUCTION}

An electrocardiogram (ECG) is a test that records the electrical activity of the heart. ECG is used to measure the rate and regularity of heartbeats as well as the size and position of the chambers, the presence of any damage to the heart and the effects of drugs or devices used to regulate the heart. The use of a simulator has many advantages in the simulation of ECG waveform. First one is saving of time and another one is removing the difficulties of taking real ECG signals with invasive and non invasive methods. This paper describes the design of a microcontroller based ECG and Heart beats monitor which outputs both normal and abnormal waveforms. Both heart rate and signal amplitude may be controlled. [1]

The ECG has a wide array of uses:

$>$ It can indicate acute or previous damage to heart muscle (heart attacks).

$>$ It is useful for the detection of conduction abnormalities.

$>$ It can be used for the monitoring of is chaemic heart disease during an stress test.

$>$ It can provide information on the physical condition of the heart. [1]

\section{NEED OF WIRELESS TRANSMISSION}

An ECG/BP signal is useful for a doctor to evaluate a patient's heart condition relating to: - Whether a heart attack has occurred - What parts of the heart is damaged - Irregular heart beats - Whether the heart is not receiving enough blood or oxygen It has been demonstrated, an ECG/BP signal is extremely valuable, making it a conventional mechanism used in hospitals by both doctors and nurses. The aim of this project is to develop a wireless system to provide a more user-friendly device for ambulatory application. Similarly it provides the doctor or nurse with a trouble-free approach to the patient's ECG/BP signal. In fact, for patients in rural and regional areas an ECG report could be sent to a doctor for examination. Thus, this is a system in which the doctors at his/her house can treat the patient in the emergency condition in the hospital. [2]

\section{WIRELESS COMMUNICATION}

Incorporation of technologies such as Bluetooth, GPRS, GSM or Wi-Fi in these systems allows wireless transmission to health or control centers. This system describes a low-cost, portable system with wireless transmission capabilities for the acquisition, processing, storing and visualization in real time of the electrical activity of the heart to a Personal Computer. The data acquisition unit, built here transmits ECG and BP signals using Bluetooth technology. Bluetooth technology were used in the system for reliable communication. Today there are still some scope in Bluetooth technology regarding coverage distance, Its growth in popularity has been due to a number of important factors. [2] 


\section{ARCHITECTURE OF WIRELESS TRANSMISSION SYSTEM}

The ECG signal is an electrical signal generated by the heart's beating, which can be used as a diagnostic tool for examining some of the functions of the heart. It has a principal measurement range of 1 to 3 $\mathrm{mV}$ and signal frequency range of 0.05 to $140 \mathrm{~Hz}$. Fig. 1 shows the block diagram of ECG module. ECG signals were acquired through a low power consumption ECG amplifier. The ECG amplifier can construct with a gain of 1000, a CMRR of 80dB and a frequency bandwidth of $0.05-100 \mathrm{~Hz}$. These signals are both amplified and filtered to the required specifications. The duration between peaks of the QRS waves (R-R intervals) can be used to calculate the beat-to-beat heart rate (HR). The output waveform from the ECG amplifier is then fed to the rest of the system. Proper ECG signal acquisition is carried out using filters for noise suppression and amplifiers to enlarge the signal amplitude as much as possible, while keeping it within the input voltage range of the analog to digital converter (ADC). The task of the ADC is then to digitize the analog voltage with a resolution high enough to represent the original signal. These values can then be collected by a microcontroller (MCU) which maintains the connection with the wireless transmitter. [3][5]

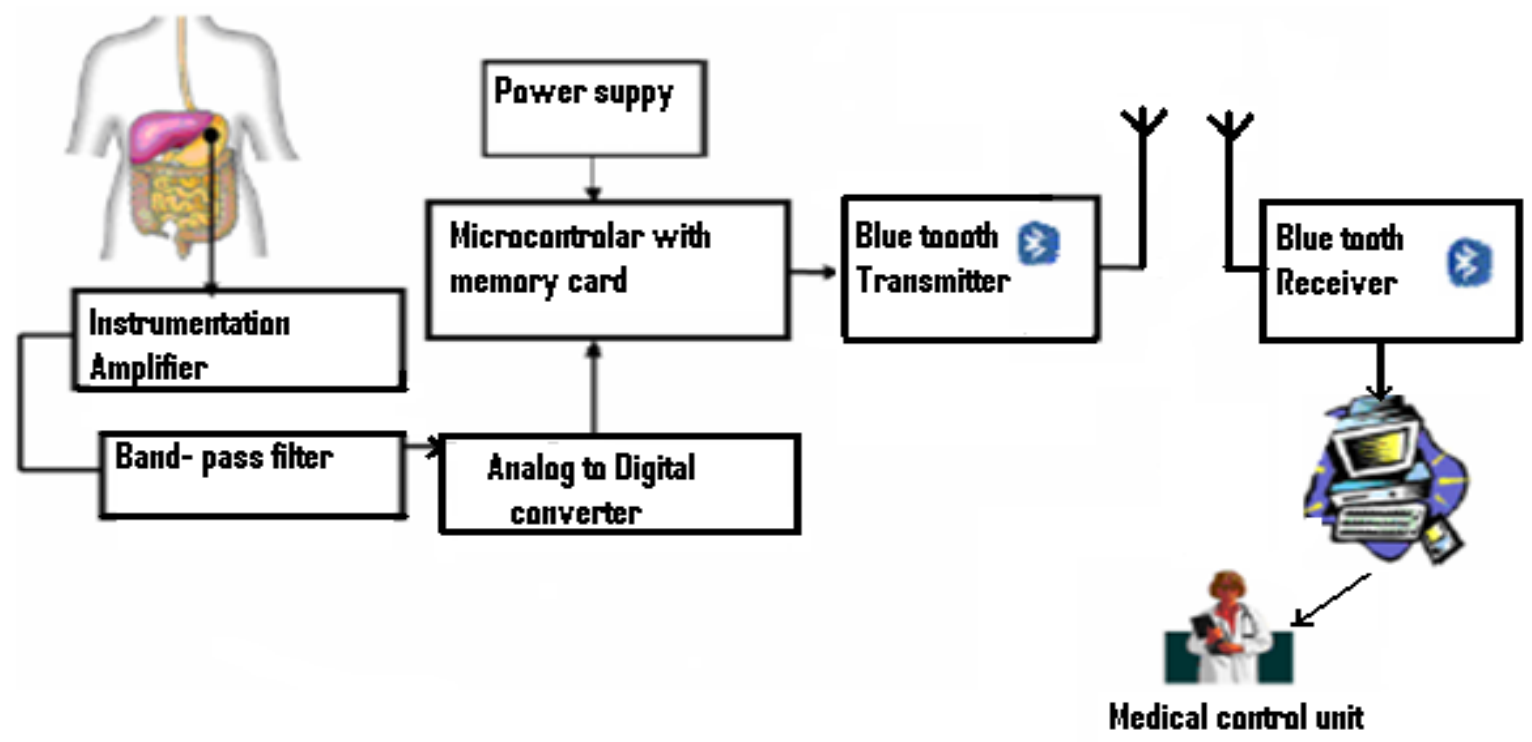

Fig. 1 Architecture of wireless transmission system

\subsection{Electrodes}

\section{CONDITIONING CIRCUIT}

Electrodes are used for sensing bio-electric potentials as caused by muscle and nerve cells. ECG electrodes are generally of the direct-contact type. They work as transducers converting ionic flow from the body through an electrolyte into electron current and consequentially an electric potential measurable by the front end of the ECG system.[3] In this project we use the piezoelectric sensor type electrode which is shown in figure 2 and piezoelectric sensor is a device that uses the piezoelectric effect to measure pressure, acceleration, strain or force by converting them to an electrical charge.[4]

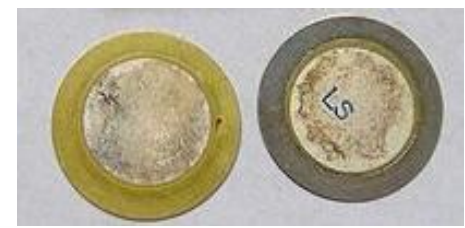

5.2 Instrumentation Amplifiers:

Figure : 2 piezoelectric electrode

The instrumentation amplifier is basically a differential amplifier that amplifies the difference between the two input signals. Hence the common mode signal is effectively eliminated. Two buffer amplifiers at the input of each signal, is provided to offer very high input impedance. The gain of the instrumentation amplifier is set around 1000. [2]

\subsection{Band pass Filter:}

To remove the unwanted frequencies in this case requires a highly selective bandpass four order filter The attenuation of low-frequency components is designed to remove the baseline (or DC) drift and obtain stable EGG recording.[2] In this project we use the VEGAKIT VK559 which is heart bit monitor circuit and one 
piezoelectric electrode connected to piezo port of this circuit. This circuit consist of IC LM386N which is basically a audio amplifier. So this circuit work as instrumentation amplifier as well as band pass filter. The circuit diagram of VEGAKIT VK559 is given below in figure 3.

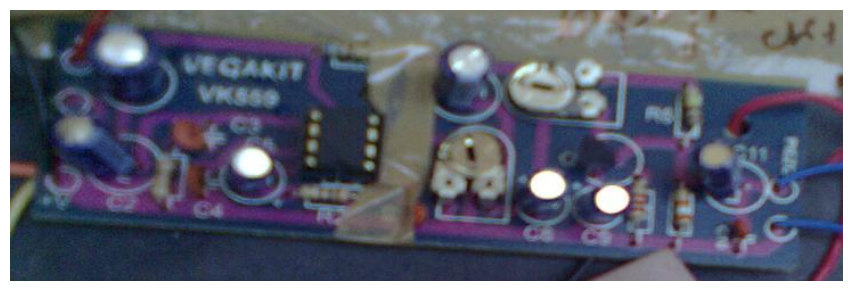

figure 3 : Amplifier circuit with electrode 1

\subsection{Microcontroller Unit:}

A Microchip 8-bit microcontroller AT89C51 by is used for outputting the values sequentially. It provides a variety of fast addressing modes for accessing the internal RAM to facilitate byte operations on small data structures. Microcontroller is used for storing the values of ECG and Blood Pressure waveforms. The AT89C51 is a low-power, high-performance CMOS 8-bit microcomputer with 4K bytes of Flash programmable and erasable read only memory (PEROM). By combining a versatile 8-bit CPU with Flash on a monolithic chip, the Atmel AT89C51 is a powerful microcomputer which provides a highly-flexible and cost-effective solution to many embedded control applications. The overall circuit diagram is given in figure 4. [4]

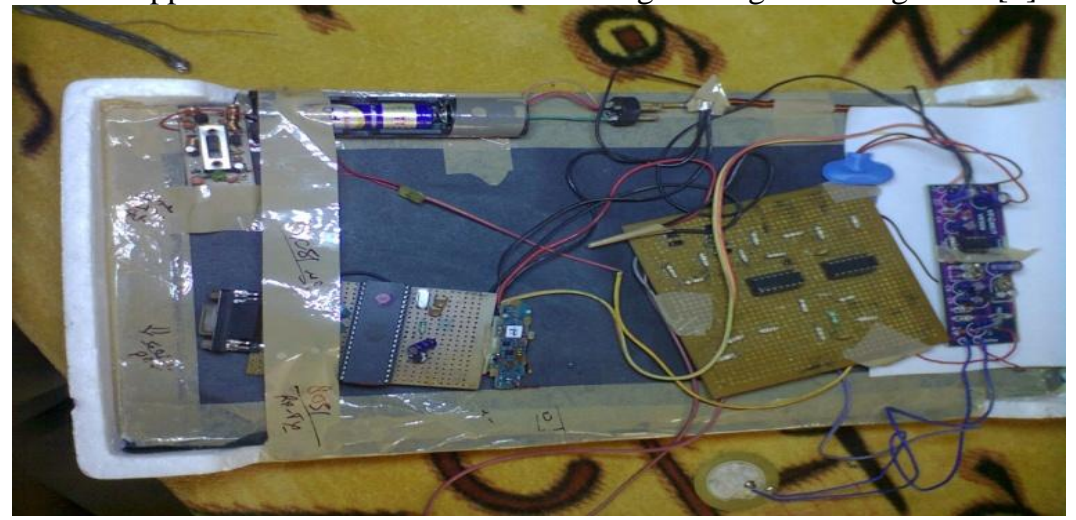

Figure : 4 Overall circuit diagram containing all parts

\subsection{The Bluetooth Transmission Module:}

Bluetooth technology is the wireless protocol used in the transmitter. Bluetooth has many advantages. First, it is available in a user-friendly modular form. There are many available Bluetooth devices which hide the Bluetooth stack and allow the user to interact with the device using simple modem commands. This reduces development time considerably. In addition, Bluetooth is a common technology on mobile phones, and this will expand the range of use for the transmitter. The Bluetooth Intelligent Serial Module HM1100 is the device selected for the wireless module. [3]

\section{ECG SIMULATION USING MATLAB}

The aim of the ECG simulator is to produce the typical ECG waveforms of different leads and as many arrhythmias as possible. My ECG simulator is a matlab based simulator and is able to produce normal lead I ECG waveform.

\subsection{Significant features of ECG waveform}

A typical scalar electrocardiographic lead is shown in Fig. 5, where the significant features of the waveform are the P, Q, R, S, and T waves, the duration of each wave, and certain time intervals such as the P-R, $\mathrm{S}-\mathrm{T}$, and Q-T intervals. 


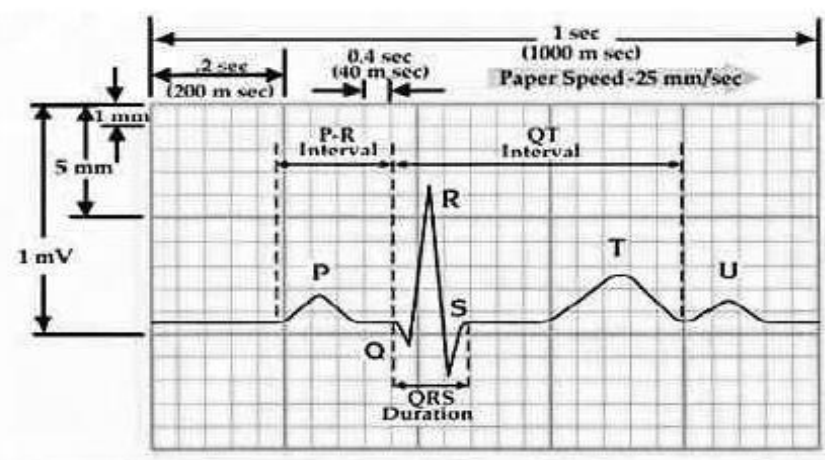

Figure : 5 Typical ECG signal

\subsection{ECG R-peaks detection algorithm development}

The basic task of electrocardiogram (ECG) processing is R-peaks detection. There are some difficulties one can encounter in processing ECG: irregular distance between peaks, irregular peak form, presence of lowfrequency component in ECG due to patient breathing etc. To solve the task the processing pipeline should contain particular stages to reduce influence of those factors. Present sample demonstrates such a pipeline. The aim is to show results of processing in main pipeline stages.

Let us have some digital ECG signal - that is our input data (fig. 6):

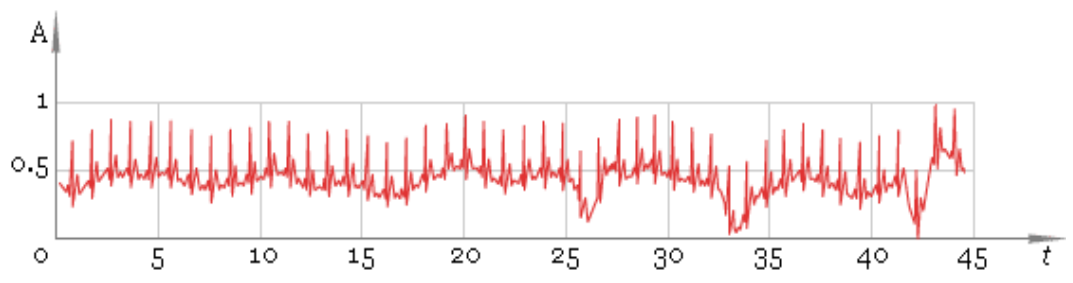

Figure : 6 Original ECG.

As one can see the ECG is uneven. Thus our first step is to straighten it. To say that in mathematical language, we should remove low-frequency component. The idea is to apply direct fast Fourier transform FFT, remove low frequencies and restore ECG with the help of inverse FFT. Here is the result of FFT processing - fig. 7.

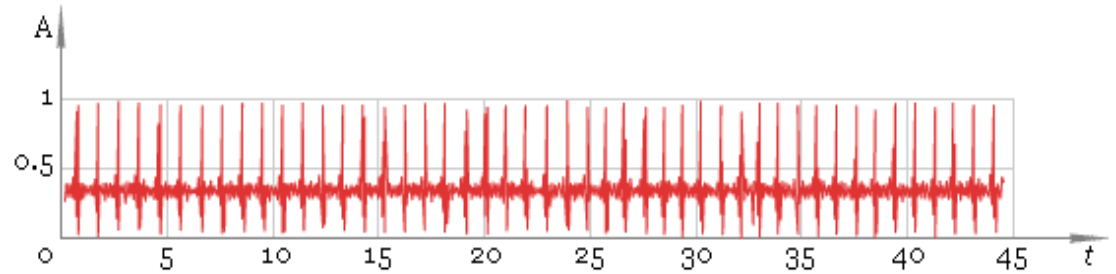

Figure : 7 FFT filtered ECG.

Our second step is to find local maxima. To do that we use windowed filter that "sees" only maximum in his window and ignores all other values. On this step we use window of default size. As result we get fig. 8 .

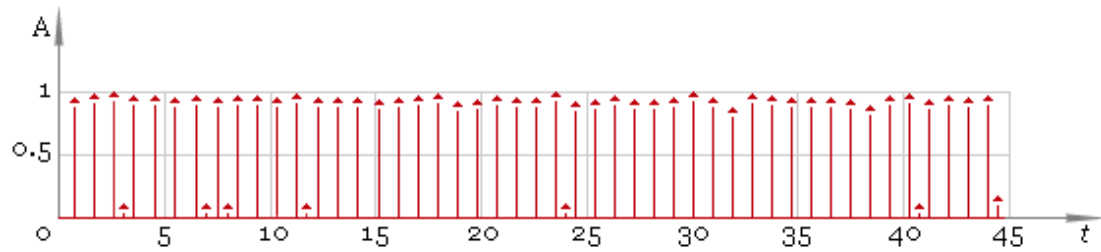

Figure : 8 Filtered ECG - first pass.

Now we should remove small values and preserve significant ones - fig. 9. Here we are using a threshold filter. 


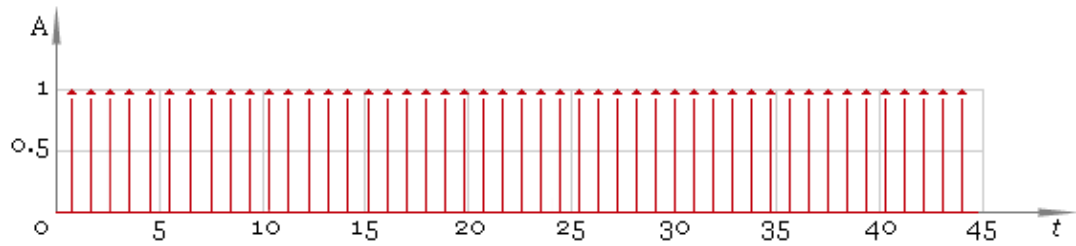

Figure : 9 Peaks.

In this case the result is good but in general case we cannot be sure we have all the peaks. So the next step is to adjust filter window size and repeat filtering - fig. 10. Compare the result with fig. 8 - now filtering quality is much better.

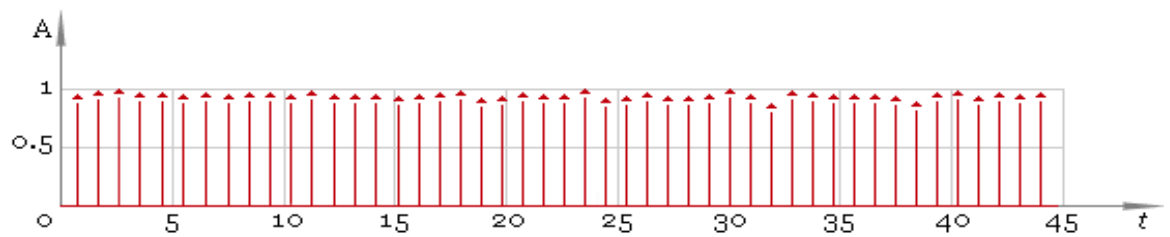

Figure : 10 Filtered ECG — second pass.

Now we are ready to get the final result and here it is: fig. 11.

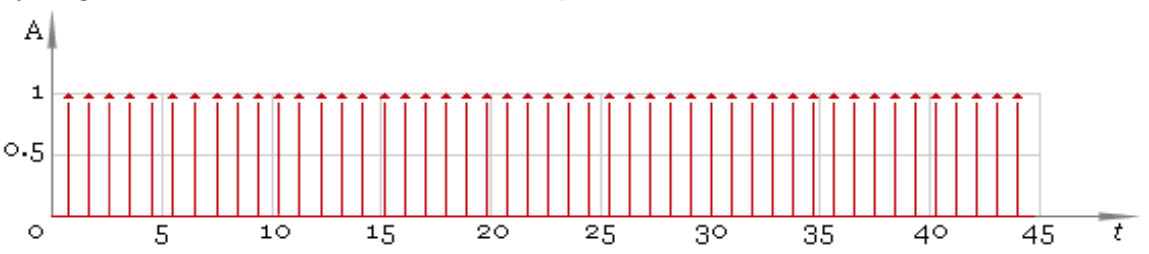

Figure : 11 Peaks - final result.

\section{ECG SIMULATION RESULT}

In this experiment an ECG waveform with standard specification like Heart beat : 72, Amplitude of $\mathrm{P}$ wave : $25 \mathrm{mv}$, Amplitude of $\mathrm{Q}$ wave : $0.025 \mathrm{mv}$, Amplitude of QRS wave : $1.6 \mathrm{mv}$, Amplitude of S wave: $0.25 \mathrm{mv}$, Amplitude of $\mathrm{T}$ wave: $0.35 \mathrm{mv}$, Amplitude of $\mathrm{U}$ wave: $0.035 \mathrm{mv}$, Duration of $\mathrm{P}$ wave : 0.09 second, Duration of Q wave : 0.066 second, Duration of QRS wave: 0.11 second, Duration of S wave: 0.066 second, Duration of T wave : 0.142 second, Duration of U wave : 0.0476 second, P-R interval : 0.16 second and S-T interval : 0.18 second taken and simulated in MATLAB then found the simulated result which is shown in figure 12 .

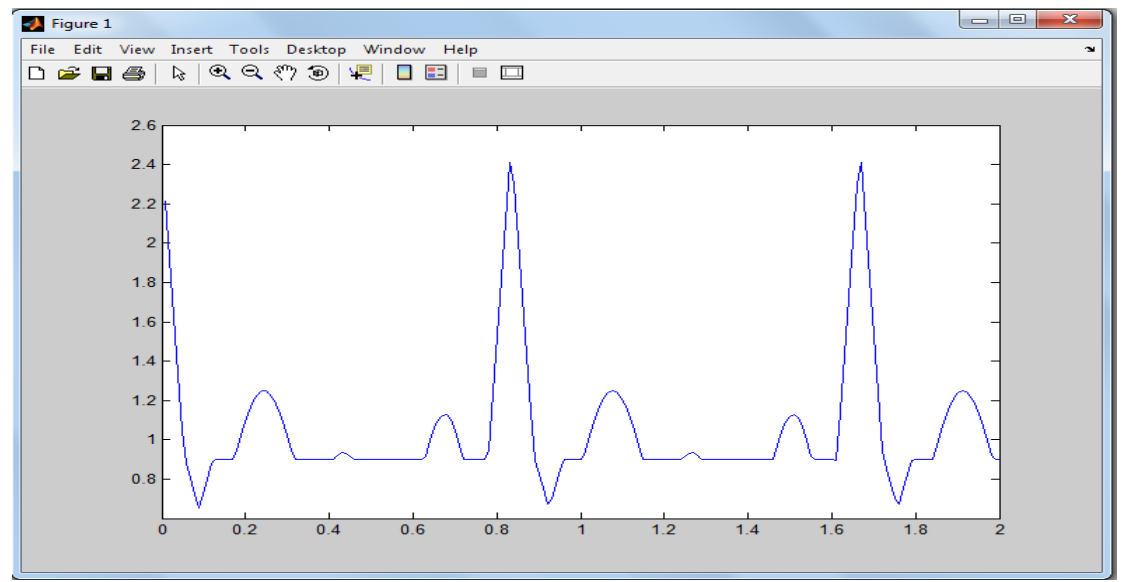

Figure : 12 Simulated ECG wave form with standard specification

Again an ECG waveform with different specification value like Heart : 90, Amplitude of $\mathrm{P}$ wave : $0.35 \mathrm{mv}$, Amplitude of $\mathrm{Q}$ wave : $0.035 \mathrm{mv}$, Amplitude of QRS wave : $1.9 \mathrm{mv}$, Amplitude of $\mathrm{S}$ wave: $0.35 \mathrm{mv}$, Amplitude of T wave: $0.40 \mathrm{mv}$, Amplitude of $U$ wave: $0.025 \mathrm{mv}$, Duration of $P$ wave : 0.10 second, Duration of Q wave : 0.065 second, Duration of QRS wave: 0.10 second, Duration of S wave: 0.065 second, Duration of T 
wave : 0.148 second, Duration of U wave : 0.0475 second, P-R interval : 0.16 second and S-T interval : 0.18 second taken and simulated in MATLAB then found the simulated result which is shown in figure 13.

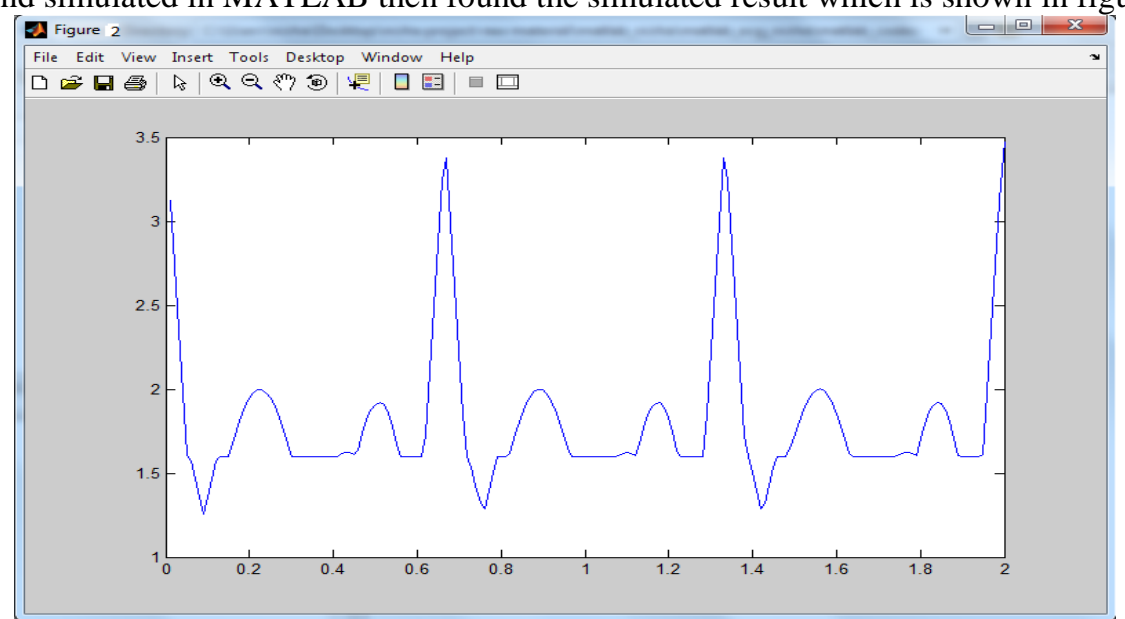

Figure : 13 Simulated ECG wave form with different specification value

With the help of different electrode we monitor the heart bit of the heart and store the heart bit sound in AT89C51 microcontroller then stored result transmitted to personal computer with the help of Bluetooth device. The Bluetooth device interfaced with MATLAB by Graphical user interface (GUI). In this work we connected the piezo electrode to the heart and monitor the heart bit sound of the heart and observed the result which is shown in figure 14 and figure 15.

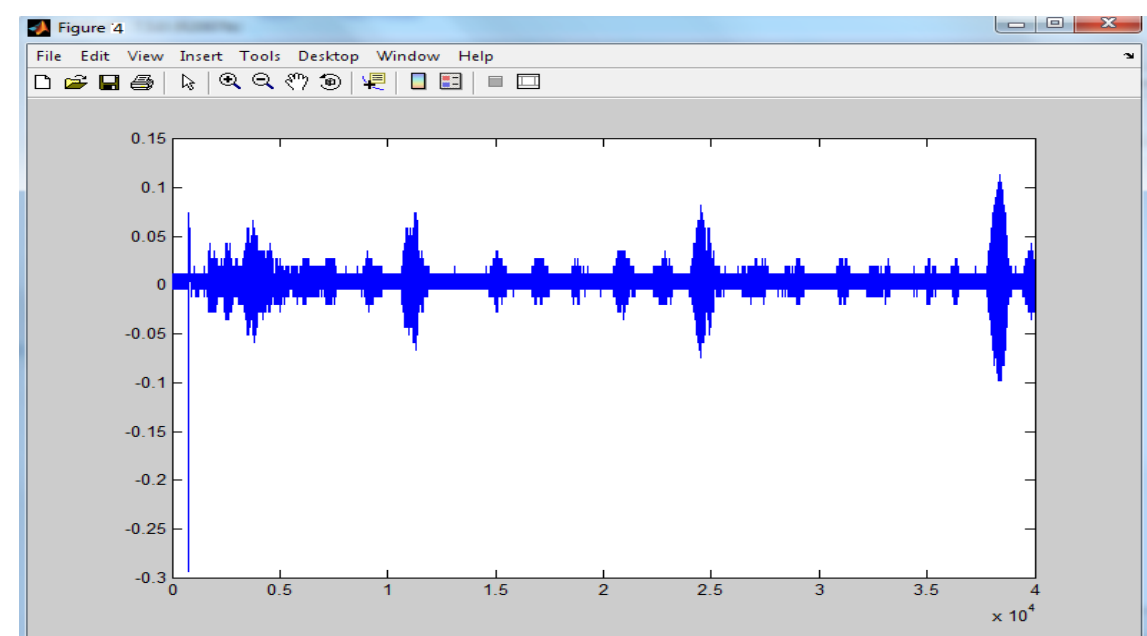

Figure : 14 Output of Heart bit monitor in first observation

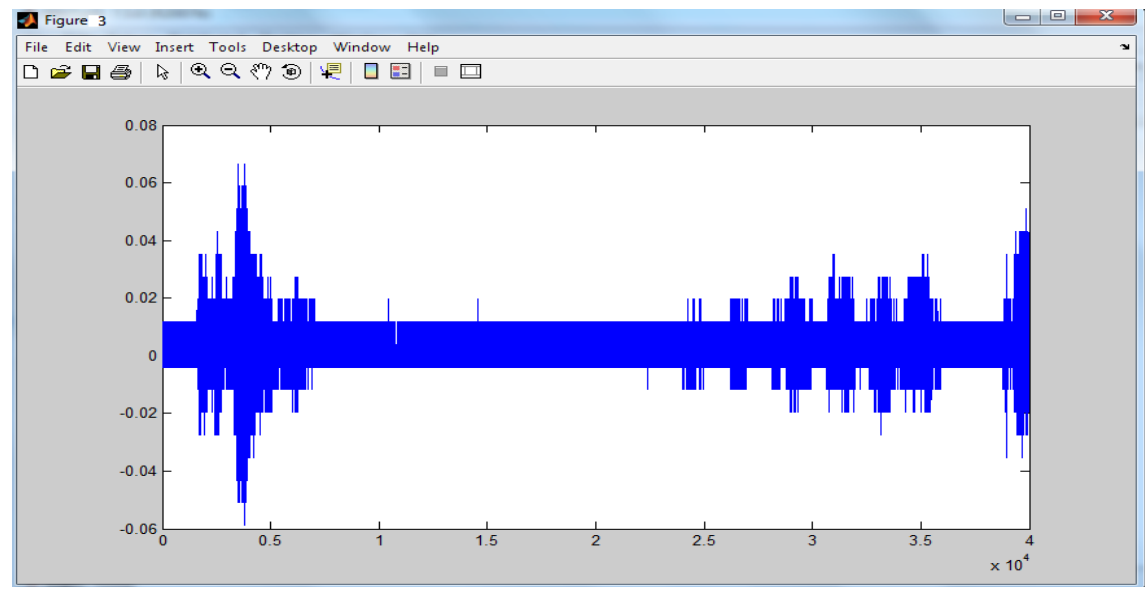

Figure : 15 Output of Heart bit monitor in second observation 


\section{CONCLUSION}

In this study, the implementation of an embedded system based on a microcontroller for real time analysis of ECG signal and then simulation of these ECG signal in MATLAB has been investigated. The system has been tested successfully on simulated ECG signals. A wireless ECG transmission system , using Bluetooth device, is able to transmit, recoard and real time display the ECG signal.

This work introduces the wireless communication electrocardiogram detection system with the use of AT89C51 microcontroller and the communication module. Here we use bluetooth device for the wireless telemedicine. So whith the help of bluetooth transmission and reception we can monitor the ECG signal of patient in the central place in any hospital.

\section{REFERENCE}

1). Nivedita Daimiwal, Asmita Wakankar, J. Instrum. Soc. India 37(4) 243-248, Cummins College of Engineering for Women, Pune - 411 052, Maharashtra.

2). Journal of Engineering Research and Studies, E-ISSN0976-7916, Swati Y.Gaikwad, Prof. Ms. Revati Shriram "Blood pressure and ECG monitoring system based on internet " Cummins college of Engg. Pune, Maharshtra, India .

3). S. haddab , M .laghrouche measurement science review, volume 9, no.5, 2009 "microcontroller -based system for electrogastrography monitoring through wireless transmission " department of electronics, mouloud mammeri university .

4). Mohamed Fezari, Mounir Bousbia-Salah, and Mouldi Bedda The international arab journal of information technology, vol 5 no. 4 oct 2008 "Microcontroller Based Heart Rate Monitor “ Department of electronics, University of Badji Mokhtar, Annaba .

5). C.-N. Chien, H.-W. Hsu Proceedings of the 2005 IEEE Engineering in Medicine and Biology 27th Annual Conference Shanghai, China, September 1-4, 2005. 